\title{
Water contents of glasshouse soils at field capacity and at saturation. 2. Estimating water contents from organic- matter and clay contents or from loss-on-ignition
}

\section{J. van den Ende}

Glasshouse Crops Research Station, P.O. Box 8, NL 2670 AA Naaldwijk, Netherlands

Received 18 January 1988; accepted 21 March 1988

Key words: water contents, glasshouse soils, field capacity, saturated soil, soil drying, organic-matter determination, clay, loss-on-ignition

\begin{abstract}
The possibilities of estimating water contents of glasshouse soils at field capacity and at saturation with the aid of organic-matter and clay contents or of loss-on-ignition values were examined. It was found that water contents of the soils at field capacity and at saturation could be accurately estimated with the use of regression equations in which loss-on-ignition was the independent variable. Multiple regression equations in which organic-matter and clay contents were the independent variables did not have a higher predictive value. The regression coefficients in equations for estimating water contents of saturated pastes obtained from air-dry soil samples amount to about $80 \%$ of the corresponding coefficients in such equations for saturated pastes obtained from field-moist soil samples. The clay fractions were found to contribute much more to the water contents of saturated soil pastes than to those of soils at field capacity.
\end{abstract}

\section{Introduction}

The water retention properties of a soil are usually determined to a high degree by its organic-matter content and texture (mainly the clay content). Consequently, specific water contents of soils, such as those at field capacity and at saturation, can often be estimated accurately by means of the organic-matter and clay contents. For certain soils, however, the nature of the organic matter (Pons \& Zonneveld, 1965) and/or clay (Odell et al., 1960; Yong \& Warkentin, 1975) and other characteristics of the soils, such as bulk density (Yong \& Warkentin, 1975) and silt or sand contents (Salter \& Williams, 1969), may have to be taken into account.

At the Glasshouse Crops Research Station, an investigation was carried out to explore the possibilities of estimating the water contents of glasshouse soils at field 
capacity and at saturation from their organic-matter and clay contents. Loss-on-ignition $\left(600^{\circ} \mathrm{C}\right)$ was also included as a means to estimate water contents. This losson-ignition is usually made up primarily of loss of organic matter and of adsorbed and structural water (Ball, 1964; Spain et al., 1982). An additional advantage is its ease of determination. The ultimate aim was to arrive at a reliable estimate of the ratio of water content at saturation and at field capacity. Knowledge of this ratio is needed when the saturation extract is to be used as the medium in which the degree of availability of nutrients is determined (van den Ende, 1988).

\section{Materials and methods}

In the investigation, use was made of soils described in a preceding paper (van den Ende, 1988), in which characteristics of the glasshouse soils were presented. The method of determination of loss-on-ignition was not mentioned in that paper. This characteristic was determined by heating a quantity of oven-dry $\left(105^{\circ} \mathrm{C}\right)$ soil at $600{ }^{\circ} \mathrm{C}$ for two hours. The resulting weight loss was then registered as mass fraction of oven-dry soil.

For convenience it is repeated in this second part of the paper (1) that the water contents of the soils at sampling time were approximately equal to those at field capacity, (2) that on the basis of their mass fractions organic matter and clay, the soils were grouped in five soil type classes, and (3) that saturated soil pastes were prepared from both field-moist and air-dry soil samples.

For water contents, expressed as mass ratios 'water/solid phase', the symbol $w$ is used. The subscripts $f$, sf and sa are used for such ratios in case of field-moist soil and of saturated soils obtained from field-moist and air-dry soil, respectively. For mass fractions organic matter, clay and loss-on-ignition of oven-dry soil, the symbols $f_{\mathrm{H}}, f_{\mathrm{C}}$ and $f_{\mathrm{I}}$ are used, respectively.

\section{Results and discussion}

\section{Water contents in relation to organic-matter and clay contents}

For the relationships between the various water contents and the organic-matter and clay contents, the following regression equations were calculated:

$$
\begin{array}{ll}
w_{\mathrm{f}}=2.630 f_{\mathrm{H}}+0.171 & r=0.971 \\
w_{\mathrm{f}}=2.374 f_{\mathrm{H}}+0.376 f_{\mathrm{C}}+0.134 & R=0.980 \\
w_{\mathrm{sf}}=3.513 f_{\mathrm{H}}+0.369 & r=0.938 \\
w_{\mathrm{sf}}=2.915 f_{\mathrm{H}}+0.876 f_{\mathrm{C}}+0.283 & R=0.965 \\
w_{\mathrm{sa}}=2.739 f_{\mathrm{H}}+0.332 & r=0.942 \\
w_{\mathrm{sa}}=2.247 f_{\mathrm{H}}+0.722 f_{\mathrm{C}}+0.261 & R=0.972
\end{array}
$$

The $w_{f}$ values appear to be closely correlated with the $f_{\mathrm{H}}$ values. Inclusion of the $f_{\mathrm{C}}$ 
values in the calculation increases the correlation coefficient only a little, although the regression coefficient of the $f_{C}$ variable in Equation 2 deviates very significantly $(P<0.001)$ from 0 . The lack of a sizeable increase in correlation coefficient can be explained by the finding that on average $w_{\mathrm{f}}$ values were determined much more strongly by $f_{\mathrm{H}}$ values than by $f_{\mathrm{C}}$ values, which is shown by the numerical values of the regression coefficients in Equation 2, and by the finding that $f_{\mathrm{H}}$ and $f_{\mathrm{C}}$ values were correlated $(r=0.573)$. The latter also implies that the contributions of the $f_{C}$ values to the $w_{f}$ values will have been included to some extent in the regression coefficient of the $f_{\mathrm{H}}$ variable in Equation 1 and that the regression coefficients of the $f_{\mathrm{H}}$ and $f_{\mathrm{C}}$ variables in Equation 2 may have been influenced somewhat by the $f_{\mathrm{C}}$ and $f_{\mathrm{H}}$ values, respectively.

Regarding the regression equations for $w_{\mathrm{sf}}$ and $w_{\mathrm{sa}}$, broadly the same conclusions can be drawn as for $w_{\mathrm{f}}$. In the case of the former equations, however, the difference between the values of $r$ and $R$ is larger. This probably results from the fact that the contributions of the $f_{\mathrm{C}}$ values to the $w_{\mathrm{sf}}$ nd $w_{\mathrm{sa}}$ values were much larger than those to the $w_{\mathrm{f}}$ values. These larger contributions are probably due to the fact that in preparing saturated soil pastes the soil was stirred. This stirring may have increased the water retention capacity of the clay fraction. It is known that mechanical energy applied to clay soil may disrupt the existing structure and may increase water retention at high pressure heads (Croney \& Coleman, 1954; Yong \& Warkentin, 1975). A proportionally large contribution of the clay fraction to the water retention capacity of soil is also found between the upper and lower plastic limits. The difference in water content between these limits is usually determined mainly by the clay content (Baver, 1940; Odell et al., 1960).

For the Equations 2, 4 and 6, the numerical value of the ratio between the regression coefficient of the $f_{\mathrm{H}}$ variable and that of the $f_{\mathrm{C}}$ variable, which is a measure of the ratio between the water retention capacities of the organic matter and the clay, is $6.3,3.3$ and 3.1 , respectively. The last two ratios agree with data reported by Pons \& Zonneveld (1965) for Dutch soils with well-humified organic matter. For such soils, these workers found a ratio value of about 3 . For soils with less humified organic matter, they found ratios varying from about 4 to 6 . Their conclusion was that the ratio is dependent on the degree of humification of the organic matter. Our data indicate that the ratio also depends on the structure of the clay fraction (see above). However, it cannot be ruled out that the pressure head in the soil influences the ratio as well.

The numerical values of the regression coefficients in Equation 6 amount to about $80 \%$ of the corresponding values in Equation 4. This indicates that the water retention capacities of both organic matter and clay decrease upon air-drying. Decreases in water retention capacity of organic matter and clay upon drying were found earlier (van Schuylenborgh, 1954; Yong \& Warkentin, 1975). Such decreases are attributable to reversible or irreversible dehydration of organic and inorganic colloidal material. The dehydration resulting from air-drying soils in our investigation was at least partly reversible. This conclusion can be drawn from experience gained during the preparation of saturated pastes. It was observed that after overnight storage of the pastes, water had to be added to many of them to re- 
store the condition of saturation. This was more the case for pastes prepared from air-dry soil than for those prepared from field-moist soil (van den Ende, 1988). It was not examined whether the rehydration process could have extended over a longer period than the duration of the overnight storage period. However, it is known that rehydration of organic matter and clay may be very slow (Baver, 1940; Hooghoudt et al., 1960).

\section{Water contents in relation to loss-on-ignition values}

The $w_{\mathrm{f}}, w_{\mathrm{st}}$ and $w_{\mathrm{sa}}$ values appeared to be correlated with $f_{\mathrm{I}}$ values too. Before the relationships between $f_{\mathrm{I}}$ values and said $w$ values are discussed, it should be remarked that $f_{\mathrm{I}}$ values were closely correlated with $f_{\mathrm{H}}$ values. For the relationship between $f_{\mathrm{I}}$ and $f_{\mathrm{H}}$, the following regression equation was calculated:

$$
f_{\mathrm{I}}=1.013 f_{\mathrm{H}}+0.019 \quad r=0.992
$$

The $f_{\mathrm{I}}$ values were higher than the $f_{\mathrm{H}}$ values; the average values amounted to 0.134 and 0.113 , respectively. This difference can, at least partly, be ascribed to the fact that in the determination of $f_{\mathrm{I}}$, in addition to the main weight loss due to destruction of organic matter, further weight loss occurs due to loss of adsorbed and structural water emanating from colloidal mineral material and to loss of $\mathrm{CO}_{2}$ from carbonate. If $f_{\mathrm{I}}$ values would be corrected for these water- and $\mathrm{CO}_{2}$ losses, the discrepancies between the two values would largely disappear. According to a correction model (unpublished) proposed by Stiboka, Wageningen, Netherlands, the water loss in question averaged a mass fraction of 0.015 , with $\mathrm{CO}_{2}$ losses being much smaller. According to data (unpublished) of our soils laboratory, these $\mathrm{CO}_{2}$ losses amount to a mass fraction of approximately 0.002 .

Differences between $f_{\mathrm{I}}$ and $f_{\mathrm{H}}$ values might also result from underestimations of $f_{\mathrm{H}}$ values. These values were obtained by multiplying organic-carbon contents by the conventional conversion factor 1.724 , and it is known that for many soils the use of this factor leads to low estimates of organic-matter contents (Broadbent, 1953; Richer \& Masson, 1964). When $f_{\mathrm{I}}$ values, corrected for water and $\mathrm{CO}_{2}$ losses mentioned above, are used as estimates of organic matter, the conversion factors for the soils used in this investigation can be calculated. Such calculations showed that for soils having $f_{\mathrm{H}}$ values higher than 0.1 , the conventional conversion factor is approximately correct. For soils having $f_{\mathrm{H}}$ values lower than 0.1 , however, the conversion factor should be close to 1.9 .

The following regression equations were found for the relationships between $f_{\mathrm{I}}$ values and the aforesaid $w$ values:

$$
\begin{array}{ll}
w_{\mathrm{f}}=2.617 f_{\mathrm{I}}+0.118 & r=0.985 \\
w_{\text {sf }}=3.537 f_{\mathrm{I}}+0.293 & r=0.964 \\
w_{\text {sa }}=2.753 f_{\mathrm{I}}+0.273 & r=0.966
\end{array}
$$

The correlation coefficients for Equations 8, 9 and 10 are practically equal to those 
for Equations 2, 4 and 6, respectively. Hence, the value of the former equations for predicting moisture contents with the use of only one independent variable $\left(f_{\mathrm{I}}\right)$ is not lower than that of the latter equations with two independent variables $\left(f_{\mathrm{H}}\right.$ and $f_{\mathrm{C}}$ ). This result will, at least partly, be due to the facts that (1) $f_{\mathrm{I}}$ values included adsorbed and structural water originating from clay particles, and (2) the value 1.724 used as conversion factor for estimating $f_{\mathrm{H}}$ values from organic-carbon contents is only approximately correct.

The relationship between $f_{\mathrm{I}}$ and $w_{\mathrm{f}}$ values is shown in Fig. 1.

Formerly, $f_{\mathrm{I}}$ values were extensively used in the Netherlands for the assessment of the water retention capacities of glasshouse soils. The capacity was calculated by

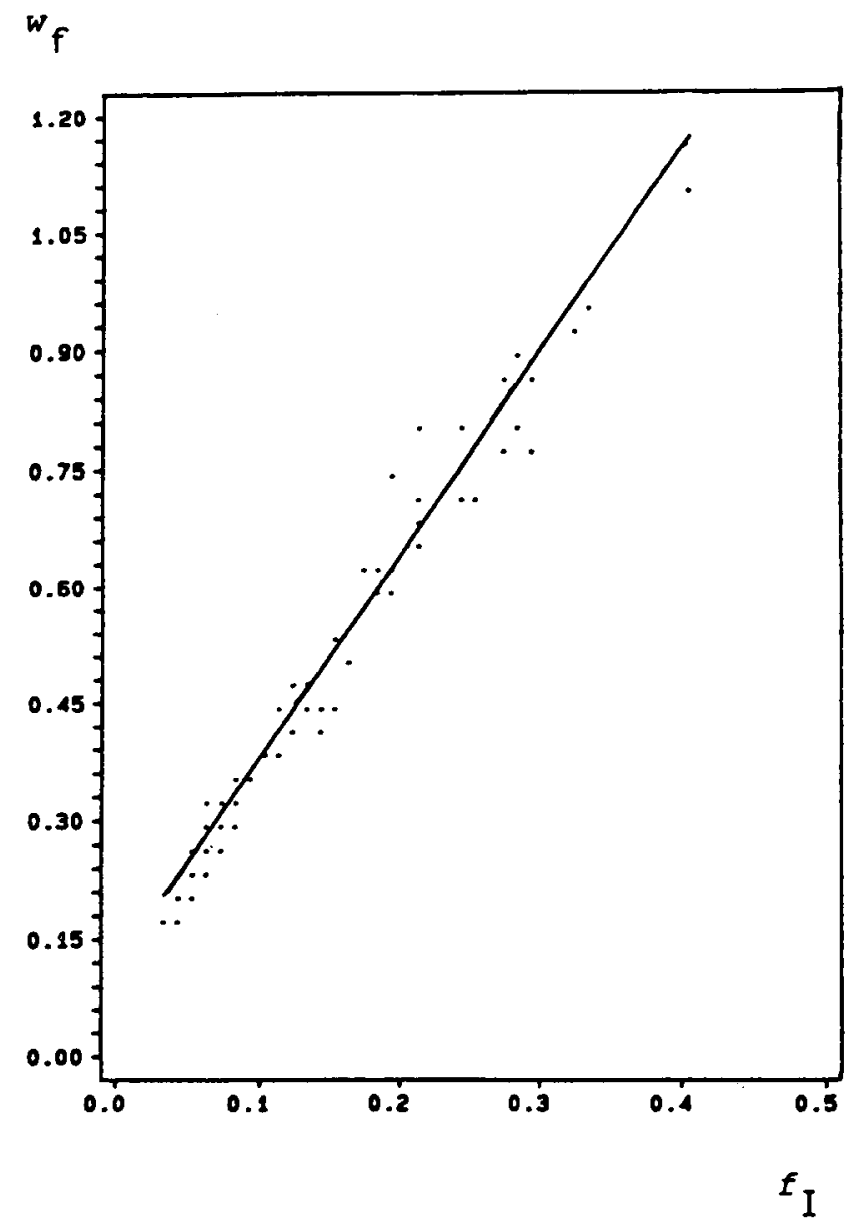

Fig. 1. Relationship between mass ratios 'water/solid phase' of field-moist soil samples $\left(w_{\mathrm{f}}\right)$ and mass fractions loss-on-ignition of the dried samples $\left(f_{\mathrm{l}}\right)$. Function adjusted:

$y=2.617 x+0.118$ $r=0.985$. 
use of the formula $2 f_{\mathrm{I}}+0.15$ (Sonneveld $\&$ van den Ende, 1971). The water contents assessed with this formula correspond rather closely with those obtained with the use of Equation 8.

\section{Water contents in relation to bulk density}

It is likely that $w$ values were influenced by factors other than water retention capacities of organic matter and clay as well. In this connection, mention must be made of the finding that the average $w_{\mathrm{f}}, w_{\mathrm{sf}}$ and $w_{\mathrm{sa}}$ values of soil type class 2 (mass fraction organic matter lower than 0.1 and mass fraction clay between 0.05 and 0.15 ) were substantially lower than those of soil type class 1 (mass fractions organic matter and clay lower than 0.1 and 0.05 , respectively), as reported by van den Ende (1988). This finding cannot be explained from differences in organic-matter and clay contents. Presumably, it was connected with the difference in particle size distribution between the two soil type classes. The particle size distributions were such that the soils in soil type class 2 will have been more prone to compaction than the soils in class 1 (Bodman \& Constantin, 1965; Faure, 1976). From bulk density values of undisturbed soil samples (van den Ende, 1988) it is apparent that in situ the soils in soil type class 2 had on average a more compact matrix geometry than those in class 1 . Such a difference in matrix geometry was probably also present under conditions of saturation. A more compact matrix geometry implies a smaller pore volume, and it is known that at high pressure heads, a smaller pore volume may result in a lower water content (Kuipers, 1961; Box \& Taylor, 1962; Hill \& Sumner, 1967). In conformity with this, the difference in water content between the soils in soil type class 1 and those in class 2 was proportionally larger for the saturated condition than for the field-moist condition.

It would have been possible to calculate a regression equation in which $w_{\mathrm{f}}$ as dependent variable and $f_{\mathrm{I}}$ and bulk density as independent variables were included. However, that calculation was not carried out because of the close correlation existing between $f_{\mathrm{I}}$ and bulk density values $(r=-0.898)$. For a similar reason, a regression equation with $f_{\mathrm{H}}, f_{\mathrm{C}}$ and bulk density as independent variables was not calculated either.

Another reason for omitting the calculations referred to is that the bulk density values of soils, particularly those of glasshouse soils, may vary in the course of time. Especially, the bulk density values of the toplayer of glasshouse soils may fluctuate strongly, with low values encountered shortly after tillage and rising values during the growing season.

\section{Conclusions}

Loss-on-ignition values of glasshouse soils were found to be closely correlated ( $r=$ 0.992 ) with organic-matter contents as estimated from organic-carbon determinations.

Water contents of the soils at field capacity and at saturation could be accurately estimated with the use of regression equations in which loss-on-ignition was the in- 
dependent variable. Multiple regression equations in which organic-matter and clay contents were the independent variables did not have a higher predictive value. This result will, at least partly, be due to the facts that (1) loss-on-ignition values included adsorbed and structural water originating from clay particles, and (2) the carbon-organic matter conversion factor value of 1.724 , used to calculate organic-matter contents from organic-carbon contents, is only an approximate value.

The numerical values of regression coefficients in the equations for estimating water contents of saturated pastes obtained from air-dry soil samples amount to about $80 \%$ of the corresponding values in such equations for saturated pastes obtained from field-moist soil samples. This finding indicates that the water retention capacities of both organic matter and clay decrease upon air-drying.

The clay fractions contributed much more to the water contents of the saturated soil pastes than to those of the soils at field capacity. Proportionally, the contributions of the organic-matter contents to each of the three water contents differed much less.

\section{References}

Ball, D. F., 1964. Loss-on-ignition as an estimate of organic matter and organic carbon in non-calcareous soils. Journal of Soil Science 15: 84-92.

Baver. L. D. . 1940. Soil Physics. 370 pp. John Wiley \& Sons, New York.

Bodman, G. B. \& G. K. Constantin, 1965. Influence of particle size distribution in soil compaction. Hilgardia 36: 567-591.

Box, J. E. \& S. A. Taylor, 1962. Influence of soil bulk density on matrix potential. Soil Science Society of America Proceedings 26: 119-122.

Broadbent, F. E. 1953. The soil organic fraction. Advances in Agronomy 5: 153-183.

Croney, D. \& J. D. Coleman. 1954. Soil structure in relation to soil suction (pF). Journal of Soil Science 5: $75-84$.

Ende, J. van den, 1988. Water contents of glasshouse soils at field capacity and at saturation. 1. Relationships between water contents. Netherlands Journal of A gricultural Science 36: 265-274.

Faure, A. . 1976. Influence de la phase argileuse sur la susceptibilité au tassement des sols. Science du Sol, No 2: 121-130.

Hill, J. N. S. \& M. E. Sumner, 1967. Effect of bulk density on moisture characteristics of soils. Soil Science 103: 234-238.

Hooghoudt, S. B., D. van der Woerdt, J. Bennema \& H. van Dijk, 1960. Irreversibly drying peat soils in the West of the Netherlands. Verslagen van Landbouwkundige Onderzoekingen No 66.23. $308 \mathrm{pp}$. Pudoc, Wageningen (in Dutch, English summary).

Kuipers, H.. 1961. Water content at pF 2 as a characteristic in soil-cultivation research in the Netherlands. Netherlands Journal of Agricultural Science 9: 27-35.

Odell, R. T., T. H. Thornburn \& L. J. McKenzie, 1960. Relationships of Atterberg limits to some other properties of Illinois soils. Soil Science Society of America Proceedings 24: 297-300.

Pons, L. J. \& I. S. Zonneveld, 1965. Soil ripening and soil classification. Publication 13, 128 pp. International Institute for Land Reclamation and Improvement, Wageningen.

Richer, A. \& P. Masson. 1964. Application de la thermogravimétrie á la détermination de la matière organique des sols. Annales Agronomiques 15: 619-632.

Salter, P. J. \& J. B. Williams, 1969. The influence of texture on the moisture characteristics of soil. 5. Relationships between particle-size composition and moisture contents at the upper and lower limits of available-water. Journal of Soil Science 20: 126-131.

Schuylenborgh. J. van, 1954. The effect of air-drying of soil samples upon some physical soil properties. Netherlands Journal of Agricultural Science 2: 50-57. 


\section{J. VAN DEN ENDE}

Sonneveld, C. \& J. van den Ende, 1971. Soil analysis by means of a 1:2 volume extract. Plant and Soil 35: 505-516.

Spain, A. V., M. E. Probert, R. F. Isbell \& R. D. John, 1982. Loss-on-ignition and the carbon contents of Australian soils. Australian Journal of Soil Research, 20: 147-152.

Yong, R. N. \& B. P. Warkentin, 1975. Soil Properties and Behaviour. 449 pp. Elsevier, Amsterdam. 